long-standing ulcerative colitis. The mucous membrane of the rectum was normal, the blood having come from haemorrhoids, and the lumen was filled with effervescing fluid faeces, which indicated that the diarrhoea was due to carbohydrate intestinal dyspepsia. Rapid recovery followed abstention from starch-containing vegetables.

\section{$X$-Ray Investigation}

The $x$ rays are very useful in investigating cases of ulcerative colitis. As the normal haustration of the bowel disappears in the affected part, it is possible, by means of a barium enema, to determine the extent of the disease. It is not uncommon for more or less of the proximal part to be spared in cases in which the pelvic colon and rectum are severely involved. When recovery takes place the haustration returns, except in very chronic cases. The proximal part of the colon is the last to be involved and the first to recover; the disease almost always begins and ends in the rectum and pelvic colon, so that sigmoidoscopy detects the earliest stage of the disease, and when the mucous membrane of the rectum and pelvic colon are found to be no longer inflamed it may be assumed that the same is true of the whole colon. A barium enema also reveals the presence of strictures, but it is possible to distinguish between those caused by prolonged spasm and those caused by organic disease only by means of an opaque meal. Narrowing due to spasm does not cause any stasis, the colon being evacuated with great rapidity, whereas that due to a fibrous stricture leads to severe stasis in the dilated proximal segment. Lastly, the presence and extent of multiple polypi can often be recognized after an opaque enema or meal.

\section{TREATMENT}

Rest in bed, warmth, and a generous mixed diet, from which the skins and pips of fruit and fibres of vegetables are alone excluded, are essential parts of treatment. When anaemia is present transfusion not only improves the general condition, but often greatly hastens the healing of the ulcers.

By absorbing gas, charcoal often makes the patient much more comfortable. Of the various drugs used locally I have found tannic acid ( 1 to 2 grains to 1 ounce) the most usefui. If given after preliminary lavage with normal saline solution under low pressure through a soft catheter introduced just beyond the anus, it reaches the caecum, and is generally just as effective as if given through an appendix or caecal stoma. Quite recently I have been using dilute hydrogen peroxide (1 drachm to 1 pint) without preliminary lavage, and I am inclined to think this may prove even more satisfactory than tannic acid.

In the first case in which I used polyvalent antidysenteric serum in 1921, the result was little short of miraculous. A young man who was almost moribund after being very ill for over a year, and in whom no improvement had followed an appendicostomy, recovered completely in a fortnight; five days after the first injection the sigmoidoscope showed that the innumerable ulcers seen a few days before had vanished, and nine days later the appearance of the mucous membrane was absolutely normal. Since then I have had a number of cases in which a dramatic recovery has taken place, and my colleagues Dr. Fawcett and Dr. Ryle, and Jerwood, Bindon Brew, and many others, have had similar experiences. Rapid recovery is most likely to occur in early cases, but it is occasionally observed in those which are very chronic. Thus, complete recovery occurred within a week in the very early case I have already mentioned in which Flexner's bacillus was isolated. More frequently the serum produces a certain amount of improvement, with the result that other treatment leads to recovery more rapidly than it would otherwise have done. In a small number of cases, especially very chronic ones, the serum appears to have no effect.

I have never seen the slightest benefit follow any form of vaccination, and in some cases the local condition has been definitely aggravated. Several American physicians have told me that they have been quite unable to confirm Bargen's enthusiastic reports about vaccination with his organism, which has not been accepted by any other American workers as the cause of ulcerative colitis.

In my experience the mortality of ulcerative colitis is extremely low. Complete recovery may occur after very long periods of treatment, and in cases which do not respond to serotherapy as much as a year or two of continuous treatment may be required. There is also an unfortunate tendency to relapse. The danger of this is much reduced if treatment is continued until the sigmoido. scope shows no trace of inflammation, even if symptoms have already disappeared for some weeks. Associated conditions, such as oral and pharyngeal infections and anal complications, must be treated, as a relapse may follow an acute sore throat or the development of a perianal abscess or a fistula in ano. The patient should always take sufficient paraffin to keep his stools soft; if achlorhydria is present, the associated gastritis should be treated, and, if the secretion of acid does not return, the patient should take hydrochloric acid for the rest of his life.

Bibliography

Bargen, J. A.: Ann. Int. Med., 1929, iii, 335.

Brew, R. Bindon: British Medical Journal, 1930, i, 478.

Dudgeon, L. S.: Lancet, 1923, i, 1007.
Eyre, J. W. H.: British Medical Journal, 1904, i, 1002.

Eyre, J. W. H.: British Medical Journ
Hawkins, H. P.: Ibid., 1909, i, 767 .

Hawkins, H. P.: Ibid., 1909, i, 767 .
Hurst, A. F.: Guy' Hospital Reports, 1921, 1xxi, 26, and Medical Essays and Addresses, 1924, 150.

Jerwood, B. E.: Guy's Hospital Gazette, 1921, xxxv, 292.

Nabarro, D.: Personal communication, 1931.

Saundby, R.: British Medical Journal, 1906, i, 1325.

Thorlakson, P. H. T.: Canadian Med. Assoc. Journ., 1928, xix, 656 ; and Trans. Amer. Proctological Soc., 1930, xxx, 100

Wills, S., and Moxon, W.: Lectures on Pathological Anatomy, second edition, 1875, p. 408 .

\section{PULMONARY TUBERCULOSIS IN CHILDHOOD*}

RY

\section{G. B. DIXON, M.R.C.S., L.R.C.P.}

CHIEF TUBERCULOSIS OFFICER, CITY OF BIRMINGHAM; MEDICAL SUPERINTENDENT OF THE CITY SANATORIUM

(With Special Plate)

I think it is fair comment to say that in the past too little attention has been paid to the diagnosis and treatment of pulmonary tuberculosis in young children. The non-pulmonary forms of this disease have received more attention and they have given more satisfactory results. The provision of beds in special institutions for pulmonary tuberculosis occurring in children has not been, in relation to the number of cases, so generous as for the nonpulmonary types ; this, and the better response to treatment of the non-pulmonary forms, have been contributory factors to the present position. In making use of the term " childhood type " of tuberculosis, I refer to lesions in the lungs and intrathoracic glands which are the result of a first infection with the tubercle bacillus.

\section{- INCIDENCE AND Course}

Figures for the year 1923, published by the National Association for the Prevention of Tuberculosis, show that in England and Wales between 2,700 and 2,800 children

* Summary of an address read to the infant welfare subgroup of the Midland branch of the Society of Medical Officers of Health. 
of from 1 to 4 years of age died from tuberculosis. Between 1,300 and 1,400 of these died from tuberculosis of the nervous system (meningitis), about 500 from tuberculosis of the intestines and peritoneum, 400 from disseminated tuberculosis, between 300 and 400 from tuberculosis of the respiratory system, and less than 100 from disease in the bones and joints. In Birmingham, during the years 1915-17, 193 cases of pulmonary tuberculosis were notified among children up to 5 years of age, and during the years $1923-25,88$ cases were notified in children of the same age ; for these periods respectively, 73 and 66 deaths occurred. Some figures dealing with the mortality rates for tuberculosis among young children in the Birmingham Children's Hospital were recently published by Smellie, ${ }^{1}$ who states that in the period 1922-29, 1.514 deaths occurred in the hospital, and that there were 1,190 necropsies, in 192 of which the cause of death was tuberculosis. He found that the highest mortality occurred among children in the second year of life, and the greatest number of deaths took place in the first four months of the year, the smallest number being recorded during August. The focus of this disease among these children was cerebral in 51 per cent. of the total number dying, abdominal in 33 per cent., and pulmonary in only 14 per cent. ; Smellie assumed that the infection was due to the human type of bacillus in no less than 64 per cent. of the series. It may be inferred from these figures either that pulmonary tuberculosis in young children is not a very common disease or that it is not always readily diagnosed.

I think it is generally accepted that pulmonary tuberculosis occurring during the first year of a child's life tends to progress rapidly, and to cause death from acute general tuberculosis or tuberculous meningitis. In the second year the disease may follow a less acute and less rapid course, but there is still little or no tendency to healing, and death usually takes place. After this period, and until the age of about 10 years, acutely progressive and rapidly fatal tuberculosis of the lungs becomes less common. Between the ages of 3 and 10 children may be found with well-marked pulmonary tuberculous lesions, which in some instances do not cause much inconvenience or give rise to any very marked or typical symptomatology. Krause ${ }^{2}$ writes:

"The more I see of tuberculosis in infants and the more I confirm the diagnosis by the aid of new methods, the more infants I find who run along with comparatively little illness."

My own experience bears out this view.

\section{Differences Between Childhood and Adult TUBERCULOSIS}

Pulmonary tuberculosis in the child has certain definite characteristics which distinguish it from the same disease in adult life ; the infantile type is the result of a primary infection, that occurring in the adult is the result of a reinfection. According to Myers, ${ }^{3}$ an area of inflammation develops where the bacilli settle in the lung, which may be small, or large enough to affect an entire lobe; from it the regional hilar and broncho-tracheal glands are quickly involved, and become enlarged. The parenchymatous lesion may heal and almost entirely disappear, a small area of calcification-that is, Ghon's body -being left (Fig. 1, Special Plate). In a comparatively short time the lymphatic glands may show calcium deposits, or they may remain caseous, without calcium deposition. In the infantile type the parenchymatous lesion may be localized in any part of the lung, least frequently in the upper lobes, and after the subsidence of the initial inflammatory changes which occur in connexion with it, may be difficult to demonstrate radio- graphically. In the adult type the lesion is apical, and the tracheo-bronchial glands are not included in the process of reinfection. Some authorities ${ }^{4}$ state that when a tuberculous process exists in any part of the body, even if remote from the lung, tubercle bacilli may be carried to the hilum of the lung, where they cause enlargement of the lymphatic glands, apart from any pulmonary parenchymatous lesion.

In the childhood type, caseous lesions tend to become calcified, and cavitation is rarer than in the adult type ; in the adult type, caseous lesions are usually associated with cavitation and fibrosis. It is well to remember in this connexion that the adult type of disease, associated with cavitation, may be found at the age of 7 or 8 years, and occasionally earlier. The distribution of the disease in young children conforms most usually to one of three types-namely, a diffuse miliary form, a localized form in which the lesion may be large or small and represented by a caseous mass with a fibrous periphery or by a massive lobar infiltration, or a broncho-pneumonic distribution. Massive distributions involving the major portions of one or both lungs are occasionally seen as terminal conditions. (See Figs. 2, 3, and 4, Special Plate.)

\section{Diagnosis}

The diagnosis of pulmonary tuberculosis in early childhood is seldom an easy matter; the physical examination of infants, who protest both vocally and physically, is not always satisfactory as far as pulmonary conditions are concerned. The information derived from percussion is never so reliable as in the case of adults, and as infants do not expectorate we are cut off from one of our more dependable sources of information. In addition, infants are usually difficult subjects for radiography; this is unfortunate, as we have to rely more upon radiographic investigation in infants suspected of pulmonary tuberculosis than we do in the case of adults. Certain special signs have been described in relation to the diagnosis of intrathoracic glandular tuberculosis in children, the better known of which are D'Espine's sign and Eustace Smith's sign ; I place slight reliance upon the evidence that their presence is supposed to furnish.

In many cases physical examination alone gives insufficient evidence upon which to base a diagnosis. The history of the case is important. Exposure to tuberculosis within the family is significant, and a history of attacks of phlyctenular conjunctivitis, of erythema nodosum, or of pleurisy is suggestive of tuberculous infection. Symptoms, as a rule, do not help us much ; they may be absent, or if present are for various reasons not always elicited. When they exist they may vary in intensity from those associated with a so-called cold to those accompanying a pneumonia. Cough, loss of weight or failure to increase weight, increased respirations, pyrexia, peevishness, and vomiting may all be present, and in the absence of discovery of other ascertainable cause should be regarded with suspicion.

\section{Radiographic Methods}

Radiography is one of the most useful and valuable methods of diagnosis at our disposal, but a negative radiographic examination should not in every instance refute a diagnosis of tuberculosis otherwise arrived at. The lesions in parenchyma or hilum may cast no shadow on the film. The hilum alone may show evidence of disease, the initial lesion being in some remote part of the body ; if in the lung, it may be obscured by the diaphragm, 11bs, or heart, and for this reason be difficult to detect, though it is usually demonstrable microscopically or inacroscopically after death. The-tracheo-bronchial glands, 
when enlarged, cannot always be demonstrated radiographically, because of their position within the mediastinum, where they are masked by the heart and large blood vessels ; but any abnormal deviation of the trachea occurring in childhood should always suggest the presence of enlarged tracheo-bronchial glands.

McPhedran ${ }^{8}$ says he

" has not seen in radiographs of the excised or living lung, non-tuberculous enlargement of the intra-pulmonary glands sufficient to allow them to be radiographically recorded.".

In this statement he refers particularly to bronchitis, broncho-pneumonia, and the chronic non-tuberculous pulmonary lesions. He also finds that

"The differentiation between the shadows cast by tuberculous hilar. glands and normal anatomical structures, such as blood vessels, is at times difficult. Homogeneous rounded or oval densities suggest vessels ruming parallel to the axial ray, particularly if somewhat symmetrical bilaterally. Although calcification in the glands of both hila is not uncommon, it is rarely that one or more do not show characteristic irregularity of coatour and inequalities of density. When densities of uncertain origin appear, decision as to their character may be facilitated by radiographing the patient again, after rotation, when, if due to the blood vessels, they will disappear."

The presence of calcium deposits in the tracheobronchial glands, or in the hilar glands, is nearly always associated with a tuberculous infection. Pleurisy probably occurs more frequently among children than is generally realized, and in many cases results from a tuberculous infection; the fibrinous type is more frequent than the serous, and it should be realized that a well-marked pleural rub may exist without any definite $x$-ray evidence of its presence. Tuberculous intrathoracic disease can often be demonstrated radiographically when it exists, if satisfactory antero-posterior and oblique films are made. It is injudicious to express a dogmatic opinion in any case if the $x$-ray examination has consisted of screening alone.

\section{Laboratory Methods of Examination}

These may provide conclusive evidence for a positive diagnosis. Smears of faeces should be examined for the presence of acid-fast bacilli, and, if these are found, an animal experiment should be carried out to ascertain if they are tubercle bacilli. Such examinations, undertaken in some hundreds of children under 8 years of age admitted to the sanatorium for observation, showed the presence of acid-fast bacilli in about 1 per cent. of the specimens examined, and in practically every instance animal experiment proved them to be tubercle bacilli. A more recent, and possibly more exact, method of finding tubercle bacilli in the digestive tract is to examine the centrifuged deposit from a gastric lavage, undertaken after the child has been fasting for six or eight hours. From $130 \mathrm{c} . \mathrm{cm}$. to $300 \mathrm{c} . \mathrm{cm}$. of sterile water are required for the purpose. The tube and glass funnel or syringe body used must be previously sterilized, as must the bottle used for collection. The fluid is centrifuged and the sediment is stained for acid-fast bacilli; some of the sediment should be plated on a suitable medium and some injected into a guinea-pig, either or both of which methods may give a positive result when suitably stained specimens of the sediment are negative.

\section{Tuberculin Tests}

For infants the two most reliable tuberculin tests ar: the quanti-Pirquet test of Morland and the intradermal test (Mantoux). Dilutions of old tuberculin, human or bovine, or both, may be used. For the former, dilutions of $1,4,16$, and 64 per cent. are used, the skin on the inner side of the forearm being a suitable site for application. After cleansing the skin, five abrasions are made, and small amounts of the progressive dilutions are applied from the wrist upwards, the fifth abrasion acting as a control. After twenty-four, and again after forty-eight, hours the arm is examined, and the diameter of any resulting papule is measured with calliper and rule. The amount of traumatic reaction is indicated by the measurement of the fifth abrasion, and this is subtracted from that of the other papules; the results are recorded after each examination and measurement. From these two readings the average papule size and the average papule difference are estimated, and are compared with Morland's modification of Erlandsen's table, ${ }^{5}$ when a unit of sensitivity can be arrived at which is useful for purposes of comparison and for records.

For the intradermal test a suitably graduated tuberculin syringe, fitted with a No. 214 gauge half-inch needle, and old tuberculin are used. In the first place, a solution of tuberculin-0.01 $\mathrm{mg}$. in $0.1 \mathrm{c} . \mathrm{cm} .-\mathrm{is}$ injected parallel to the surface, into the superficial layers of the skin. If no reaction results in twenty-four to ninety-six hours, the procedure is repeated with a solution of which 0.1 c.cm. contains $0.1 \mathrm{mg}$. of tuberculin ; in the absence of reaction within five days, the test is repeated with a solution of which $0.1 \mathrm{c} . \mathrm{cm}$. contains $1 \mathrm{mg}$. of tuberculin. If no reaction occurs after any of these, it may be assumed that the child has not been infected. When a positive reaction occurs, it is usually evident within twenty-four to forty-eight hours, appearing as a deep red nodule surrounded by a pink halo. A tuberculin reaction when positive cannot be accepted alone as evidence for the necessity of treatment; it merely indicates that the person has been infected, and to be infected has not the same significance as to be affected, although infants giving a strong positive reaction without evidence of active or latent tuberculosis should always be kept under supervision and examined from time to time. If reaction occurs and is associated with latent tuberculosis, it is advisable to keep the child under constant supervision, and to advise such prophylactic measures as extra nourishment and admission to an open-air school. By latent tuberculosis $^{6}$ I mean a lesion which is unaccompanied by symptoms evident to patient or friends, or by physical signs recognizable by the physician, and which is detected only by tuberculin reactions and by characteristic changes shown on $x$-ray films. In relation to the various tuberculin tests, it should be recalled that in grave tuberculosis the test may be negative, and sensitivity may be reduced or absent after pneumonia, enteric fever, influenza, pertussis, or measles, and in other conditions affecting adults, among which is pregnancy.

Reviewing the various points upon which we may depend for a diagnosis, I would rely upon the followi:!g factors:

1. The presence of physical signs and symptoms.

2. Definite involvement of the parenchyma, lung lilum, or both, as revealed by $x$-ray examination.

3. A positive reaction to a tuberculin test.

4. The demonstration of tubercle bacilli.

5. A history of exposure within the family.

The first and fifth may be very difficult to establish, and their absence should never be regarded as conclusive evidence in ruling out a diagnosis of tuberculosis. The fourth, too, is not always positive, and although conclusive when present, is no more a sine qua non than the presence of a positive sputum in adults. A positive tuberculin test and a positive $x$-ray examination, when other causes for the condition in the lung have been ruled out, may be accepted as sufficient for a positive diagnosis in many cases. ${ }^{7}$

\section{Prophylaxis}

The incidence and death rates for tuberculosis among adolescents are high in this country, and are said to be so because the " infantile period," as far as tubercle is con- 
cerned, is the " neglected period" from the point of view of diagnosis and treatment. In the past we have given much attention to the isolation and palliative treatment of large numbers of chronic infectious cases of tuberculosis in Birmingham, removing the patients from their homes to hospital pavilions in sanatoriums, in an endeavour to prevent spread of infection among other members of the family. This procedure has possibly influenced the accelerated decrease in the tuberculosis mortality rate which has occurred during recent years in Birmingham. The percentage decrease ${ }^{12}$ between the five years 1883 to 1887 , and a similar period 1898 to 1902 , was 4.15 , while the percentage decrease between the five years 1903 to 1907, and a similar period 1918 to 1922 , was 24.24 .

In my opinion, rigorous and compulsory segregation of all infective cases would in many instances be a harsh procedure, and might defeat the end in view; voluntary segregation, however, practised benevolently and secured by tactful and sympathetic persuasion, should be aimed at. If this is to succeed, the patients with advanced disease should not be exposed to the open air to the same extent as ambulant patients, and, so far as visitors and recreations are concerned, they may with advantage be granted concessions which would not be given to more robust patients with early disease. The object is to get them into the sanatorium for long periods as often as possible when they are infective, unable to work, and ineffectively isolated at home. The larger the percentage of deaths from pulmonary tuberculosis occurring in these hospital pavilions the better for the community. This alone is insufficient to break the vicious circle of tuberculosis infection within the family, unless at the same time vigorous measures are adopted to diagnose and treat tuberculosis in infancy and early childhood, which would be facilitated if the departments dealing with infant welfare and tuberculosis co-operated more closely. The infant welfare departments would help greatly by passing on to the tuberculosis department definite and suspected cases of tuberculosis, or any infants who gave a positive tuberculin reaction, in order that both the infant and the family of which it was a member might be investigated. A positive tuberculin reaction in an infant is frequently the first indication of the presence of tuberculosis in the family circle, ${ }^{13}$ and the suspected child is often the first link in a chain of events leading to the diagnosis of tuberculosis in a parent. Measures designed to prevent the spread of tuberculosis can only be regarded as efficient if the family is treated as the unit for investigation.

The number of contacts which can be examined in any clinic depends entirely upon the size of the medical staff, but a majority of the cases dealt with should be children, and they should be kept under supervision for long periods, with re-examination at intervals, and the investigation should always include an $x$-ray examination and a tuberculin test. Arrangements should be made to prevent, as far as possible, continuous exposure of the contact child to infection. Co-operation between the two departments might also be extended to provide residential accommodation for the ante-natal and maternity care of pregnant women suffering from pulmonary tuberculosis. After the puerperium, these patients should be admitted to sanatoriums for prolonged periods, and their infants might be placed with advantage in a pavilion attached to a babies' hospital, which would fulfil the requirements of a "preventorium." Such arrangements would mean additional expenditure, but if they helped to lessen an appreciable amount of infantile infection and subsequent adult disease they would be justified.

The use of pasteurized milk ${ }^{9}$ has been shown to diminish the mortality rate from non-pulmonary tuberculosis between the ages of 1 and 4 years, but has little effect upon the mortality rates during the first year of life. The first infection of a young child with tuberculosis is said by some authorities to confer a certain amount of immunity, but this is not always sufficient to withstand massive doses and continuous exposure. If the primary tuberculous infection of a child is not overwhelming and lethal in its effect, a condition of latent tuberculosis may result. Opie ${ }^{10}$ suggests that latent tuberculosis increases resistance and occasionally affords complete protection from subsequent disease; it is nevertheless a source of danger, because it may be transformed into active progressive disease, and any immunity it confers should be regarded as limited and transient only. In adults ${ }^{11}$ who show no scars of an infantile infection, and who subsequently develop tuberculosis, the disease assumes the characteristics of childhood infection, and may pursue a rapidly fatal course. This has been observed particularly among the negro races, and suggests that infantile infection confers some immunity.

\section{Production of Immunity}

In recent years attempts have been made to produce immunity in infants by the inoculation of tubercle bacilli that have been attenuated in virulence. Calmette and his fellow worker Guérin have practised this extensively. The method adopted is to give by the mouth $1 \mathrm{cg}$. on the third, fifth, and seventh days of life. If a positive tuberculin reaction does not result, they suggest giving onefortieth to one-twentieth of a centigram of the vaccine subcutaneously. Where this is done a positive tuberculin reaction is obtained within six to eight weeks. About 200,000 infants are reported to have been treated in this way. Calmette's views as to the freedom from danger of this method of treatment have not been universally accepted. Fears have been expressed that an avirulent strain of bacilli, when injected into the human body, might regain its virulence.

\section{Conclusions}

In our attempts to prevent tuberculosis we cannot emphasize too strongly or too frequently that it is a communicable disease, and that it is therefore a family disease; when an adult in the family suffers from the disease and no precautionary measures are taken, it has been shown that he may infect 50 per cent. or more of the younger members of the family. It follows, therefore, that the family as a whole, and not the patient alone, should be the unit for investigation and continued supervision. Children with active disease should be given prolonged periods of treatment in a sanatorium in which provision is made for the schooling of those whose condition allows of it. Children with latent tuberculosis, and those who give a positive reaction to the tuberculin test, should be kept under supervision for prolonged periods. Everything possible should be done to provide them with adequate food and clothing, and to remove them from continuous exposure to infection in the home.

The radiographs illustrating this article were taken by $\mathrm{Mr}$. W. H. Smith.

REFERENCES

1 Smellie: Birmingham Med. Rev., v, 1.

2 Krause: Decline of Mortality from Tuberculosis Among Infants and Children, Boston Med. and Surg. Journ., 1926, June.

3 Myers: Tuberculosis Among Children.

4 Krause: Amer. Rev. of Tuberculosis, 1925, xi, 303

${ }^{5}$ Riviere: Early Diagnosis of Tubercle.

- Opie: Infection with Tuberculosis in Children and Adults, Journ. of Outdoor Life, 1927, xxiv, 274.

"Myers: Tuberculosis Among Children.

8 Myers: Tuberculosis Among Children.

${ }^{8}$ McPhedran: Henry Phipps Institute,

10 Opie: Infection with Tuberculosis in Children and Adults, Journ. of Outdoor Life, 1927, xxiv, 274.

${ }^{11}$ Idem: Spread of Tuberculosis within Families, Journ. Amer. Med. Assoc., 1926, lxxxvii.

${ }^{12}$ Philip, Sir R.: British Medical Journal, 1931, i, 43.

13 Myers: Tuberculosis Among Children. 


\section{G. B. DIXON : PULMONARY TUBERCULOSIS IN CHILDHOOD}

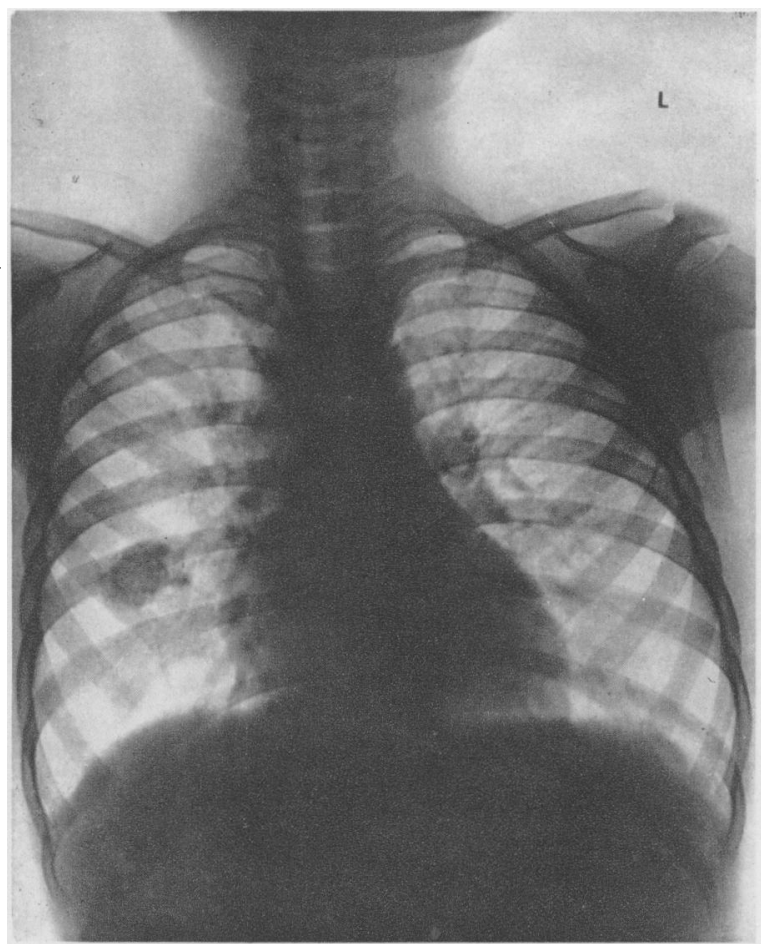

Fro. 1.-Female aged 7. Father died pulmonary tuberculosis, sputum poaltive. Pirquet test positive. Large Ghon's tuberclo right base. Filiar diveane both sides.

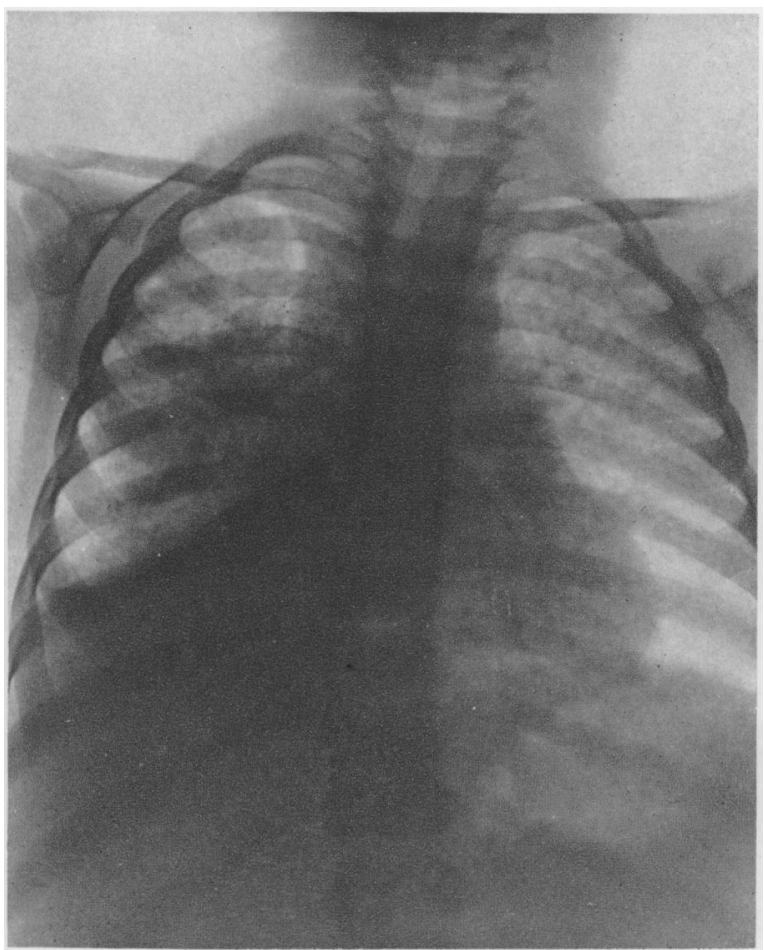

Fra. 3.-Female aged 4. Mother suffering from pulmonary tuberculoais with positive sputum. Opacity over base of right lung extending upwards along mediastinal margin. Miliary mottling throughout large part of both lungs, conglomerate in right mid area.

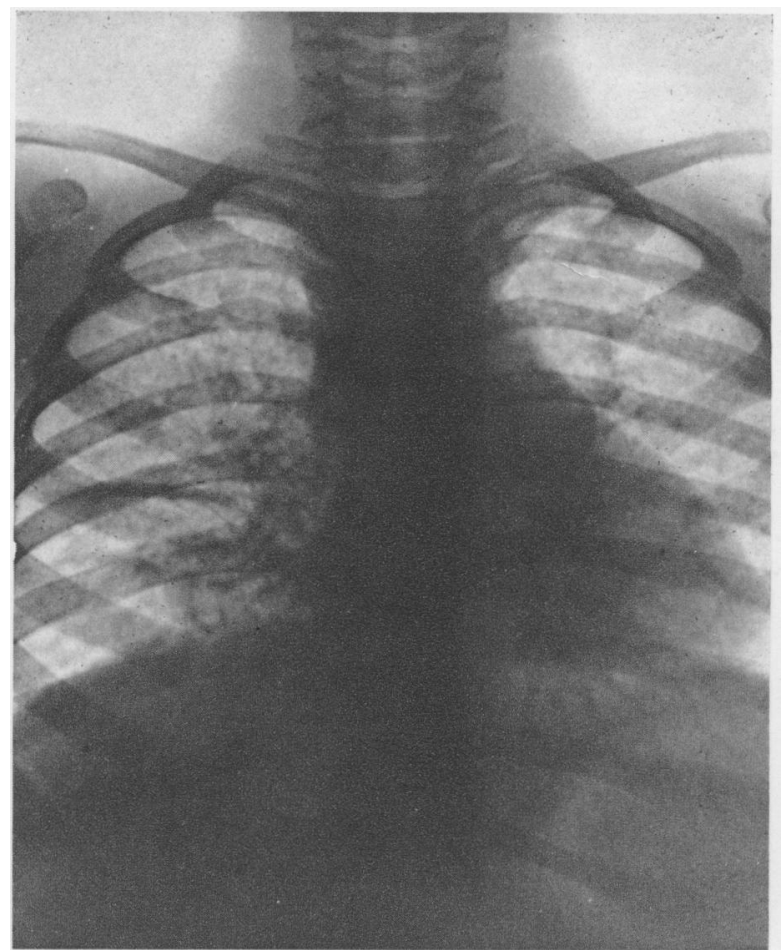

Fre. 2.-Female aged 31. No known family history of tuberculosis. Film shows flocenlent mottling in both lungs, confluent in right cardiophrenic area. Thickening of right lower interlobar septum. Miliary distribution.

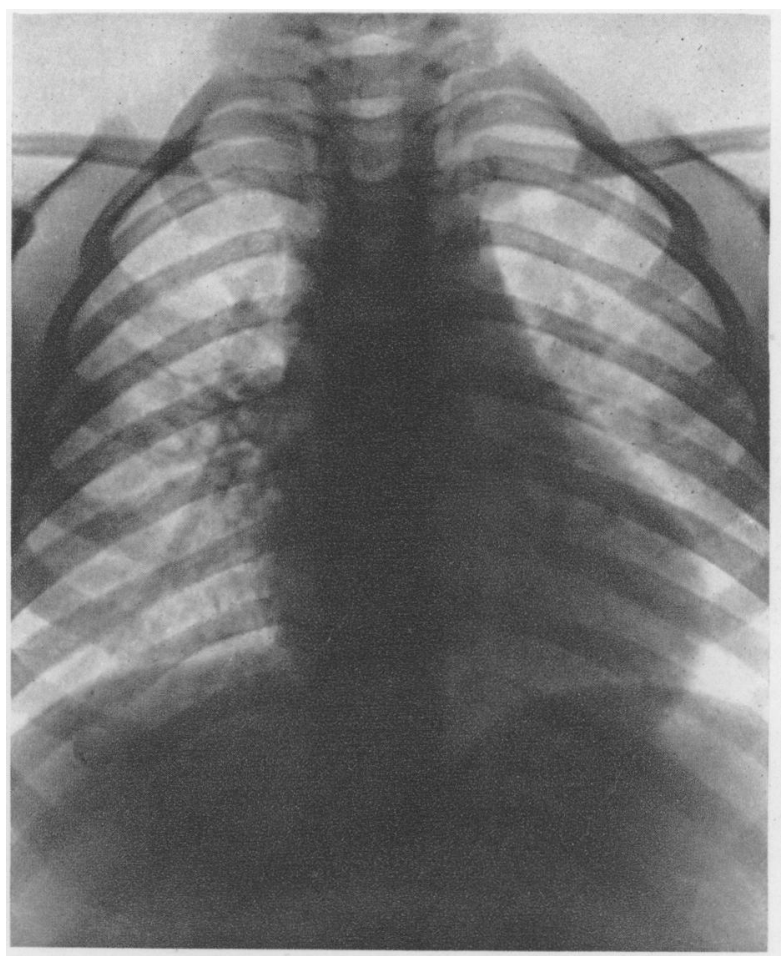

Fra. 4.-Female aged 5. Father suffering from pulmonary tuberculosis, sputum positive. Irregular glandular mass at right hilum. Inflitration of both perihilar zones. 